\title{
Vector field theories in cosmology
}

\author{
A. Tartaglia and N. Radicella \\ Dipartimento di Fisica del Politecnico and INFN section of Turin \\ Corso Duca degli Abruzzi 24, I-10129 Torino, Italy \\ e-mail: angelo.tartaglia@polito.it and ninfa.radicella@polito.it
}

October 25, 2018

\begin{abstract}
Recently proposed theories based on the cosmic presence of a vectorial field are compared and contrasted. In particular the so called Einstein aether theory is discussed in parallel with a recent proposal of a strained space-time theory (Cosmic Defect theory). We show that the latter fits reasonably well the cosmic observed data with only one, or at most two, adjustable parameters, whilst other vector theories use much more. The Newtonian limits are also compared. Finally we show that the CD theory may be considered as a special case of the aether theories, corresponding to a more compact and consistent paradigm.
\end{abstract}

\section{Introduction}

The most successful field theories of the XXth century are in general tensor theories on a four-dimensional manifold. This is true for the electromagnetic field as well of course as for the gravitational interaction. In the former case the "root" of the theory is in a four-vector potential, in the latter also the potential is a rank 2 symmetric tensor (the metric tensor).

Although, properly speaking, "tensor" includes any rank from 0 (a scalar) up to higher values, by "tensor" theory one normally means a theory based on an at least rank 2 tensor. On this respect we shall here discuss "vector" theories as rank 1 tensor theories.

Theories of this sort have not been considered as frequently as scalar and scalar-tensor theories. Of course we need a motivation and ours has been at the cosmic scale. Since 1998 evidence has been found pointing at an accelerated expansion of the universe (see [1) and theorists have been working since then to find an explanation of the alleged behaviour. In various forms a sort of dark energy fluid has been envisaged (see 2] and [3]) or modifications of the classical General Relativity theory have been proposed producing the sought for effects (see [4] and [5]). One of us has put forth the idea that a cosmological vector field be responsible for the acceleration: let us call the one based on this vector, "Cosmic Defect" (CD) theory [6]. 
Another cosmic vector field has been discussed in the literature in recent years in a group of theories that we shall call here, for short, Vector Æther Theories (VET), although their authors have used different names or no name at all. One of these theories has indeed been christened Einstein Æther (Æ) 7 . In all cases by "æther" the cosmic vector field is meant.

The initial motivation for the VET was not related to cosmology, but rather to fundamental quantum field theory, where reasons exist to doubt of an exact Lorentz invariance. In fact, the Lorentz group is non-compact and leads to divergences in quantum field theory, associated with states of arbitrary high energy and momentum. Furthermore, because of the non-compactness of the group, it is not possible to experimentally test the invariance at all scales of energy.

Considering a $D$-dimensional universe with $D-4$ compactified extra-dimensions, the so-called tensor-induced Lorentz symmetry breaking has been investigated in [8, and a general discussion of the purely gravitational and cosmological aspects has been made.

Many other approaches to field theory, like non-commutative field theory [9] as well as non-string approaches to quantum gravity ( see references in [8]) suggest Lorentz violation.

In practice the presence of a vector field in a four dimensional space-time selects locally a special direction (in [8] also the possible breaking in the extradimensions is discussed). If the vector is time-like the local Lorentz symmetry is somehow broken and this has been the starting point for VET [10. Of course, once the global existence of a vector field has been hypothesized, its presence will bring about consequences also at the cosmic scale and here is where an intersection with the CD theory is found.

Actually, since the '70's a vector theory of gravity was proposed, somehow generalizing the Brans-Dicke theory, in which gravitation was produced by a rank-2 tensor field and a scalar field. The authors in [11] looked for detectable effects due to the motion of the solar system relative to a preferential reference frame and discussed both cosmological and solar system constraints.

The local Lorentz symmetry breaking should however not be overestimated. A cosmic privileged reference frame actually exists: the one of the cosmic microwave background radiation $(\mathrm{CMB})$ or, which is the same, in any Robertson Walker (RW) universe (as ours is commonly assumed to be, on the average) the co-moving frame of the cosmic "fluid" of galaxies. This local selection of a privileged time axis, or the equivalent Lorentz symmetry breaking, shows up only on cosmic time scales when the effects of the expansion appear. Locally, in usual time scales nothing is manifested even though the "privileged" frame is there, unless possibly for phenomena at very high energies.

Leaving these comments on the background the points on which one would like to gauge a theory are:

- ability to reproduce a given observed behaviour;

- absence of unwanted "side effects";

- simplicity; 
- existence of a reasonable interpretation frame or paradigm for the theory to fit in.

On these points we shall elaborate throughout the paper. In Sec. [II] we shall review the VE theories, inspecting the Lagrangian and deriving the equations of motion; in Sec. III the same will be done with the CD theory. In the following two sections the results of both the VET and the CD theory will be analyzed in two respects: in Sec. IV the cosmological solution will be derived, looking for an accelerated expansion; in Sec. $\mathrm{V}$ the Newtonian limit is discussed and in both cases the Poisson equation is recovered with a rescaling of the coupling constant. In Sec. VI we shall show that the CD theory may be viewed as a special case of the VET and the Standard Model Expansion (SME) theory, and finally we shall draw some conclusions (Sec. VII).

\section{Vector Æther Theories}

Adopting the traditional approach to field theory, we may write the total action integral for a space time containing a vector field $U$ as the sum of three parts

$$
S=\int \frac{d^{4} x}{c} \sqrt{-g}\left(\frac{1}{2 \kappa} R+\mathcal{L}_{U}+\mathcal{L}_{m}\right) .
$$

Of course $g$ is the determinant of the metric tensor and $R$ is the scalar curvature of the manifold. $\mathcal{L}_{U}$ is the Lagrangian density of the vector field, and $\mathcal{L}_{m}$ is the one of matter; $\kappa=\frac{8 \pi G}{c^{4}}$ is the coupling constant between matter and geometry. Writing the action in the form (11) we are implicitly assuming that no direct coupling between the vector field $U$ and matter exists; both the vector field and matter couple with geometry.

According to [12] and [13], the most general form for the Æther Lagrangian density in the action integral (1) for a vector-tensor theory, including terms up to second order in derivatives and fourth order in the fields, is written as follows:

$$
\mathcal{L}_{U}=K_{\mu \nu}^{\alpha \beta} \nabla_{\alpha} U^{\mu} \nabla_{\beta} U^{\nu}-k\left(U^{\mu} U_{\mu}-M^{2} n^{\alpha} n_{\alpha}\right)^{2},
$$

We can recognize a kinetic and a potential term for the vector field. The coefficients of the kinetic term are contained in the rank 4 tensor:

$$
\begin{aligned}
K_{\mu \nu}^{\alpha \beta} & =K(1)_{\mu \nu}^{\alpha \beta}+K(2)_{\mu \nu}^{\alpha \beta}, \\
K(1)_{\mu \nu}^{\alpha \beta} & =c_{1} g^{\alpha \beta} g_{\mu \nu}+c_{2} \delta_{\mu}^{\alpha} \delta_{\nu}^{\beta}+c_{3} \delta_{\nu}^{\alpha} \delta_{\mu}^{\beta}+c_{4} U^{\alpha} U^{\beta} g_{\mu \nu}, \\
K(2)_{\mu \nu}^{\alpha \beta} & =c_{5} \delta_{\nu}^{\alpha} U^{\beta} U_{\mu}+c_{6} g^{\alpha \beta} U_{\mu} U_{\nu}+c_{7} \delta_{\mu}^{\alpha} U^{\beta} U_{\nu}+c_{8} U^{\alpha} U^{\beta} U_{\mu} U_{\nu},
\end{aligned}
$$

in which the terms in $c_{4}$ and $c_{8}$ represent directional covariant derivatives along the field $U^{\mu}$.

The potential term in (2) is the gravitational analogue of the Higgs mechanism of gauge theories, so that the vector field acquires a vacuum expectation value $M n^{\alpha}$ that breaks the Lorentz invariance; $n$ is a unit four-vector. The action integral written with (2) is slightly different from the one that appears 
in [12] and [14 because of the different choice made for constants. Assuming $U$ to be dimensionless, one is left with dimensionless $c_{i}$ 's and $M$, too. Notice, as already stressed before, that the matter Lagrangian couples only with the metric and not directly with the $U^{\mu}$ 's.

The claim of generality on (2) must be taken with some caution, because it depends on a number of limiting assumptions on space-time. Within a wider framework and in the attempt of constructing a theory in which both General Relativity and the Standard Model are taken into account, Konstelecký has developed SME (Standard Model Extension), a theory whose effective Lagrangian contains the fields of the Standard Model as well as gravity together with additional Lorentz symmetry-violating terms. The most general formulation of SME [15] uses an Einstein-Cartan background, including torsion; in this framework (2) appears as a special subclass of Lagrangians. An earlier version of the SME in a Minkowski spacetime had already been studied in [16].

Konstelecký and Samuel in [8], as early as in 1989, considered a Lagrangian density which now could be seen as a subclass of the VE theories. It has been put forth again by Jacobson and Mattingly [10] in 2001, in practice considering the only $K(1)$ term of (3) and replacing the potential term by a constraint on the norm of the vector field, introduced by means of a Lagrange multiplier $\lambda$. The Lagrangian density for the vector field is then 1

$$
\mathcal{L}_{U}=K(1)_{\mu \nu}^{\alpha \beta} \nabla_{\alpha} U^{\mu} \nabla_{\beta} U^{\nu}+\lambda\left(U^{\alpha} U_{\alpha}-1\right) .
$$

From now on we shall refer to this theory as Einstein Æther (Æ), from the name used in [10].

The case analyzed in the earlier formulation corresponds to choosing all parameters to be zero except for $c_{1}$ and $c_{3}$, for which $c_{1}+c_{3}=0$ holds.

A theory equivalent to this choice for the Æther Lagrangian had already been studied by Nambu [17] in the case of Minkowski spacetime, who proved it to be equivalent to electrodynamics in a non-linear gauge. Other contributions in the non-flat background case are found in [10] and [18.

A variant of $\mathbb{E}[19]$ introduces the vector field in the action in the form of a function $\mathcal{F}$ of the scalar $\mathcal{K}$ obtained from the $K(1)_{\mu \nu}^{\alpha \beta}$ after choosing $c_{4}=0$ :

$$
\begin{aligned}
\mathcal{K} & =M^{-2} K_{\mu \nu}^{\alpha \beta} \nabla_{\alpha} U^{\mu} \nabla_{\beta} U^{\nu} \\
K_{\mu \nu}^{\alpha \beta} & =c_{1} g^{\alpha \beta} g_{\mu \nu}+c_{2} \delta_{\mu}^{\alpha} \delta_{\nu}^{\beta}+c_{3} \delta_{\nu}^{\alpha} \delta_{\mu}^{\beta} .
\end{aligned}
$$

This approach was motivated by its authors, Zlosnik, Ferreira, and Starkman (ZFS, for short) by the quest of a modified Newtonian gravity at galactic scales, as an alternative to dark matter. The Lagrangian for the Æther is now written $(19])$

$$
\mathcal{L}_{U}=M^{2} \mathcal{F}(\mathcal{K})+\lambda\left(U^{\mu} U_{\mu}-1\right)
$$

assuming that the Lagrange multiplier $\lambda$ has the dimension of the inverse of a squared length. Of course (5) coincides with the $Æ$ Lagrangian when $\mathcal{F}(\mathcal{K}) \equiv \mathcal{K}$.

${ }^{1}$ We shall use a +--- signature throughout the paper. 
In the general Lagrange density (2), $U^{\mu}$ is neither restricted to have a fixed norm nor to be timelike. In the case of a homogeneous and isotropic universe2, however, the assumed space isotropy implies the vector field to be timelike

$$
U^{\mu}=(U(t), 0,0,0)
$$

and still leaves six free parameters in the equations of motion [12]: $c_{2}, c_{3}, c_{4}$, $c_{7}, c_{8}$, and $k$.

This freedom is somewhat reduced in [7, [10, [19] and in the analysis made by Carroll [20] and Lim [21. All these authors constrain the vector field to be a unit vector

$$
U^{\mu}=(1,0,0,0)
$$

and maintain four (see [7] 10, 22]), or three (see 19] 20, 21]) free parameters, like in (4) and (5).

Varying (11) with respect to the metric tensor elements, we obtain, as usual, the Einstein equations in the form

$$
G_{\alpha \beta}=\kappa\left(T_{\alpha \beta}^{U}+T_{\alpha \beta}^{m}\right),
$$

where $T_{\alpha \beta}^{m}$ is the stress-energy tensor for matter, while $T_{\alpha \beta}^{U}$ is the one of the vector field. In the case of the $Æ$ theory, the explicit form of $T_{\alpha \beta}^{U}$ is

$$
\begin{aligned}
T_{\alpha \beta}^{U} & =\frac{1}{2} \nabla_{\sigma}\left[\mathcal{F}^{\prime}\left(J_{(\alpha}^{\sigma} U_{\beta)}-J_{\alpha}^{\sigma} U_{\beta)}-J_{(\alpha \beta)} U^{\sigma}\right)\right] \\
& +c_{1} \mathcal{F}^{\prime}\left[\left(\nabla_{\nu} U_{\alpha}\right)\left(\nabla^{\nu} U_{\beta}\right)-\left(\nabla_{\alpha} U_{\nu}\right)\left(\nabla_{\beta} U^{\nu}\right)\right]+\frac{1}{2} g_{\alpha \beta} M^{2} \mathcal{F}+\lambda U_{\alpha} U_{\beta}
\end{aligned}
$$

where

$$
\begin{aligned}
\mathcal{F}^{\prime} & =\frac{d \mathcal{F}}{d \mathcal{K}} \\
J_{\sigma}^{\alpha} & =\left(K_{\sigma \gamma}^{\alpha \beta}+K_{\gamma \sigma}^{\beta \alpha}\right) \nabla_{\beta} U^{\gamma} .
\end{aligned}
$$

Varying the action with respect to $U^{\mu}$, under the same hypotheses, one obtains the equations of motion for the vector field

$$
\nabla_{\alpha}\left(\mathcal{F}_{\beta}^{\prime \alpha}\right)=2 \lambda U_{\beta}
$$

\section{The Cosmic Defect theory}

The CD theory, as the theories mentioned in the previous section, ascribes the behaviour of the universe as a whole to the presence of a cosmic four-vector field. The difference with respect to the VE theories is mostly in the motivation and interpretation of the vector, then in the choice of the initial Lagrangian density for space time.

\footnotetext{
${ }^{2}$ The line element is of the form $d s^{2}=c^{2} d t^{2}-a(t)^{2} \delta_{i j} d x^{i} d x^{j}$.
} 
The CD vector field is interpreted in terms of a paradigm considering the space time as a continuum hosting a defect. The presence of defects (in the sense of Volterra [23]) in a medium implies a permanent state of stress and strain even in the absence of applied forces from outside. If we think of the Robertson Walker symmetry, assumed to be correct for describing the universe, we find an initial singular state giving rise to the symmetry. In four dimensions we may think as if we had a pointlike defect at the origin (big bang) inducing a strain everywhere in space tim 3 . A vector field naturally arises from this view: the "radial" rate of stress $\gamma$ induced by the defect [6]. Now "radial" means along the cosmic time axis.

The mentioned identification of the vector field implies it to be divergencefree, which means

$$
\gamma_{; \mu}^{\mu}=0
$$

In the RW symmetry and adopting a co-moving cosmic reference frame, (9) has the solution

$$
\begin{aligned}
& \gamma^{0}=\frac{Q^{3}}{a^{3}} \\
& \gamma^{i}=0
\end{aligned}
$$

where $Q$ is an integration constant and $a$ is the scale factor of the universe. In fact the time component of the vector is also a measure of its norm

$$
\chi^{2}=\left(\gamma^{0}\right)^{2}
$$

This result introduces a first difference with respect to the $\rightleftarrows$ theory because there the cosmic four-vector is constrained to have unit norm, in fact coinciding with the four-velocity of an observer co-moving with the cosmic fluid. This is not the case of the general Lagrangian density (2), where there is no fixed norm constraint.

As for the choice of the Lagrangian density, the CD theory uses another analogy based on the remark that the phase space of a RW universe is bidimensional and that it formally coincides with the one describing a point particle moving across a viscous fluid. Starting from this formal correspondence the action integral for the only space time is assumed to be [6]:

$$
S=\frac{1}{2 c \kappa} \int e^{-g_{\mu \nu} \gamma^{\mu} \gamma^{\nu}} R \sqrt{-g} d^{4} x
$$

where $\gamma$ is again the already mentioned four-vector.

In a RW symmetry the action integral, including matter (Friedman Robertson Walker case), reduces to

$$
\begin{aligned}
S & =S_{g}+S_{m} \\
& =\mathcal{V}_{k}\left[-\int \frac{3}{\kappa} e^{-Q^{6} / a^{6}}\left(a^{2} \ddot{a}+a \dot{a}^{2}\right) d \tau+\kappa_{0} \int f a^{3} \dot{a}^{2} d \tau+\varpi \int h a^{3} d \tau\right](12)
\end{aligned}
$$

\footnotetext{
${ }^{3}$ In the case of a spatially flat spacetime we should rather refer to a singular surface than to a singular event, but the logic structure remains the same.
} 
where now $\tau$ is the cosmic time $(=c t)$; dots denote cosmic time derivatives; $\mathcal{V}_{k}$ is the part of the Lagrangian which is not affected by any variation with respect to the metric; $\kappa_{0}$ and $\varpi$ are appropriate coupling constants; $f$ and $h$ are scalar functions of $a$ accounting for anything we could widely speaking dub as "matter".

We remark that the second derivative of $a$ with respect to $\tau$ appears linearly in the Lagrangian (the integrand of (12)). This means that integrating the corresponding term by parts in the action leads to

$$
\begin{aligned}
\int e^{-Q^{6} / a^{6}} a^{2} \ddot{a} d \tau= & \\
& \left.e^{-Q^{6} / a^{6}} a^{2} \dot{a}\right|_{\tau_{1}} ^{\tau_{2}}-2 \int e^{-Q^{6} / a^{6}}\left(3 \frac{Q^{6}}{a^{5}}+a\right) \dot{a}^{2} d \tau .
\end{aligned}
$$

One is then left with a surface term, whose variation is by definition zero, and a first order derivative term so that in practice the effective Lagrangian becomes

$$
\mathcal{L}=\frac{3}{\kappa} e^{-Q^{6} / a^{6}}\left(6 \frac{Q^{6}}{a^{5}}+a\right) \dot{a}^{2}+\kappa_{0} f a^{3} \dot{a}^{2}+\varpi h a^{3} .
$$

It is now possible to write the Hamiltonian density function for the system. This is:

$$
\begin{aligned}
\mathcal{H} & =\dot{a} \frac{\partial \mathcal{L}}{\partial \dot{a}}-\mathcal{L} \\
& =\left[\kappa_{0} f a^{3}+\frac{3}{\kappa} e^{-Q^{6} / a^{6}}\left(6 \frac{Q^{6}}{a^{5}}+a\right)\right] \dot{a}^{2}-\varpi h a^{3}
\end{aligned}
$$

and is, as usual, interpreted as the energy density (in the universe). We may easily verify that

$$
\frac{d \mathcal{H}}{d \tau}=0
$$

We may then write

$$
\left[\kappa_{0} f a^{3}+\frac{3}{\kappa} e^{-Q^{6} / a^{6}}\left(6 \frac{Q^{6}}{a^{5}}+a\right)\right] \dot{a}^{2}-\varpi h a^{3}=\mathcal{W}=\text { constant } .
$$

Then the field equation becomes

$$
\dot{a}^{2}=\frac{\varpi h a^{3}+\mathcal{W}}{\left[\kappa_{0} f a^{3}+\frac{3}{\kappa} e^{-Q^{6} / a^{6}}\left(6 \frac{Q^{6}}{a^{5}}+a\right)\right]} .
$$

Actually, if we want to recover the usual meaning of the matter term in a co-moving reference frame we must choose

$$
\kappa_{0}=0
$$

so the rate of expansion equation becomes: 


$$
\dot{a}^{2}=\frac{\kappa}{3} \frac{\varpi h a^{3}+\mathcal{W}}{e^{-Q^{6} / a^{6}}\left(6 \frac{Q^{6}}{a^{5}}+a\right)} .
$$

In the absence of a defect, it would be (FRW universe)

$$
\frac{\dot{a}^{2}}{a^{2}}=\frac{8 \pi G}{3 c^{4}} \rho c^{2}=\frac{\kappa}{3} \rho c^{2}
$$

Evaluating (15) with $Q=\chi=0$, that is looking at the equation that comes from the action of the CD theory but in the absence of a defect, we obtain

$$
\frac{\dot{a}^{2}}{a^{2}}=\frac{\kappa}{3} \frac{\varpi h a^{3}+\mathcal{W}}{a^{3}} .
$$

Of course the value of the $\mathcal{W}$ constant depends on the type of space-time we consider: in the classical empty case (no defect, no matter) it would be $\mathcal{W}=0$. We then conclude that in order to recover the classical result, i.e. comparing (16) and (17), it must be

$$
\begin{aligned}
\varpi & =1 \\
h & =\rho c^{2}
\end{aligned}
$$

where now $\rho$ represents the usual mass-energy density function.

The final expansion rate equation is

$$
\dot{a}^{2}=\frac{\kappa}{3} \frac{\rho c^{2} a^{3}+\mathcal{W}}{e^{-Q^{6} / a^{6}}\left(6 \frac{Q^{6}}{a^{5}}+a\right)}
$$

Introducing the new variable $\tilde{a}=a / Q$ and using $Q$ also as the unit for time $(\tau \rightarrow \tau Q)$ we may recast (18) in the form

$$
\dot{a}^{2}=\frac{\tilde{\kappa}}{3} \frac{\tilde{\rho} c^{2} \tilde{a}^{3}+\mathcal{W}}{6+\tilde{a}^{6}} \tilde{a}^{5} e^{1 / \tilde{a}^{6}}
$$

where the coupling constant $\kappa$, as well as the volume entering the definition of $\rho$, have been rescaled on $Q$ :

$$
\tilde{\kappa}=\frac{\kappa}{Q} \quad ; \quad \tilde{\rho}=Q^{3} \rho .
$$

\section{The accelerated expansion}

\section{In the Vector Æther theories}

Let us investigate, now, cosmological solutions deduced from the VE theories, i.e. from (6). As we already know, in the case of a homogeneous and isotropic universe the constraints of ZFS and Æ theory force the four vector field to be $U^{\mu}=(1,0,0,0)$. The energy-momentum tensor for matter, thought of as a 
perfect fluid, can be written as $T_{\alpha \beta}^{m}=\rho c^{2} u_{\alpha} u_{\beta}+p\left(g_{\alpha \beta}-u_{\alpha} u_{\beta}\right)$, where $\rho c^{2}$ is the energy density, $p$ is the pressure and $u^{\mu}$ is the four velocity of the fluid (i.e. $\left.g_{\alpha \beta} u^{\alpha} u^{\beta}=1\right)$. Equation (8) for the vector field can be used to deduce $\lambda$ and put it in the stress-energy tensor for $U^{\mu}$, eq.(17), so that one is left with the two Einstein equations:

$$
\begin{aligned}
H^{2} & =\kappa\left(\alpha H^{2} \mathcal{F}^{2}-\frac{1}{6} \mathcal{F} M^{2}\right)+\frac{\kappa}{3} \rho c^{2} \\
-H^{2}-2 \frac{\ddot{a}}{a} & =-\kappa\left[\mathcal{F}^{\prime} \alpha\left(2 H^{2}+\frac{\ddot{a}}{a}\right)-\dot{\mathcal{F}}^{\prime} \alpha H+\frac{1}{2} \mathcal{F} M^{2}\right]+\kappa p,
\end{aligned}
$$

where $\alpha$ is a combination of the $c_{i}$ 's, namely $\alpha=c_{1}+3 c_{2}+c_{3}$ and $H \equiv \frac{\dot{a}}{a}$. The equations (20) 4 govern the evolution of the universe. An accelerated expansion is indeed obtained choosing, for an appropriate range of $\mathcal{K}$ values, 19

$$
\mathcal{F}(\mathcal{K})=C(-\mathcal{K})^{n}
$$

where $C$ is a constant and $n$ an integer. $\mathcal{K}$ is found to be $3 \frac{\alpha H^{2}}{M^{2}}$.

Restrictions on the $c_{i}$ 's are obtained when studying the consistency of the theory in the perturbative regime, that is performing classical perturbations in flat space time, and at a quantum level, when the Hamiltonian has to be positive definite. The analysis has been performed by Lim in 21 in the case of the $Æ$ theory; the Lagrangian for the Æther turns out to have a scalar-type and a vector-type perturbation (i.e. a spin- 0 mode and a spin- 1 mode). The constraints obtained by Lim are the following:

- $c_{1}<0$, in order to insure that spin-0 states have positive norm, i.e. are non-ghost-like;

- $0 \leq \frac{c_{1}+c_{2}+c_{3}}{c_{1}} \leq 1$, in order to have a well-behaved propagation of the spin-0 mode;

- $c_{1}+c_{3} \geq 0$ in order to have gravitational waves propagating subluminally.

All these conditions together imply

$$
\begin{aligned}
c_{1} & <0 \\
c_{2} & \leq 0 \\
c_{1}+c_{2}+c_{3} & \leq 0
\end{aligned}
$$

i.e., $\alpha \leq 0$; this is the reason why $\mathcal{K}$ appears with a minus $\operatorname{sign}$ in $\mathcal{F}$ in (21). In [19] it is also shown that one can rewrite the Einstein equations to obtain, with the particular choice of $\mathcal{F}$ written above in (21):

$$
\left[1+\epsilon\left(\frac{H}{M}\right)^{2(n-1)}\right] H^{2}=\frac{\kappa}{3} \rho c^{2},
$$

\footnotetext{
${ }^{4}$ There is a slight difference between the equations written here and those found in [19, because of the definition of the Lagrangian for the Æther: $\mathcal{L}_{U}$ here is $16 \pi G / c^{4}$ times the one found in the cited article.
} 
where $\epsilon=-(1-2 n) C(-3 \alpha)^{n} / 6$. This solution introduces two more completely free parameters, besides the ones already present in the Lagrangian. It is then possible to choose them so that $\epsilon<0$ and find out that $H$ tends to the attractor: $\tilde{H}=M(-\epsilon)^{1 / 2 n(1-n)}$. Besides the $c_{i}$ 's, $C$ and $n$, the mass scale $M$ is also present; the authors (ZFS) relate it to the acceleration scale $a_{0}$ of Milgrom's MOND theory 24, in order to have the right limit at galactic scale.

Restricting to $Æ$ case (cfr. [10]) the equations reduce to

$$
\begin{aligned}
H^{2} & =\kappa \alpha H^{2}+\frac{\kappa}{3} \rho c^{2} \\
-\left(H^{2}+2 \frac{\ddot{a}}{a}\right) & =\kappa\left[-\alpha\left(H^{2}+2 \frac{\ddot{a}}{a}\right)+p\right] .
\end{aligned}
$$

The analysis of these equations has been performed by Carroll and Lim in [20], but their $\alpha$ parameter is opposite in sign with respect to the one used here, because we are following the notations of [19]; furthermore in their case $\kappa \alpha$ is a dimensionless quantity.

Inspecting the first equation in (22), which is the 00-th component of the Einstein equations, one can easily see that the contribution from the stress-energy tensor of the vector field is proportional to the square of the Hubble parameter. In practice the equations can be rewritten as the usual Friedmann equations just rescaling the gravitational constant $G$ :

$$
\begin{aligned}
H^{2} & =\frac{\kappa}{3(1-\kappa \alpha)} \rho c^{2} \\
\frac{\ddot{a}}{a} & =-\frac{4 \pi G_{c}}{3 c^{4}}\left(\rho c^{2}+3 p\right),
\end{aligned}
$$

The effective gravitational constant $G_{c}$ is

$$
G_{c} \equiv \frac{G}{1-8 \pi G \alpha / c^{4}} .
$$

Since $\alpha \leq 0$ the effect of the vector field is to increase the rate of expansion of the universe, but $G$ is not directly measurable. In order to obtain constraints on $\alpha$ values, one has to consider other situations, first of all the Newtonian limit.

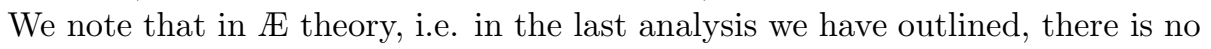
accelerated expansion, since it retraces the GR solution.

\section{In the CD theory}

The interesting feature of eq. (18) is that it does indeed contain an accelerated expansion phase in the history of the universe. Studying the properties of (18) we see that the expansion rate starts with an infinite value at the origin and tends to 0 at infinity. The initial expansion is exponential, i.e. inflationary; at the other end, for any reasonable behaviour of matter, the expansion continues for ever at a rate asymptotically tending to 0 .

If the defect is a property of space-time the expansion (which is our way to describe what actually is a static state in four dimensions) is present even 


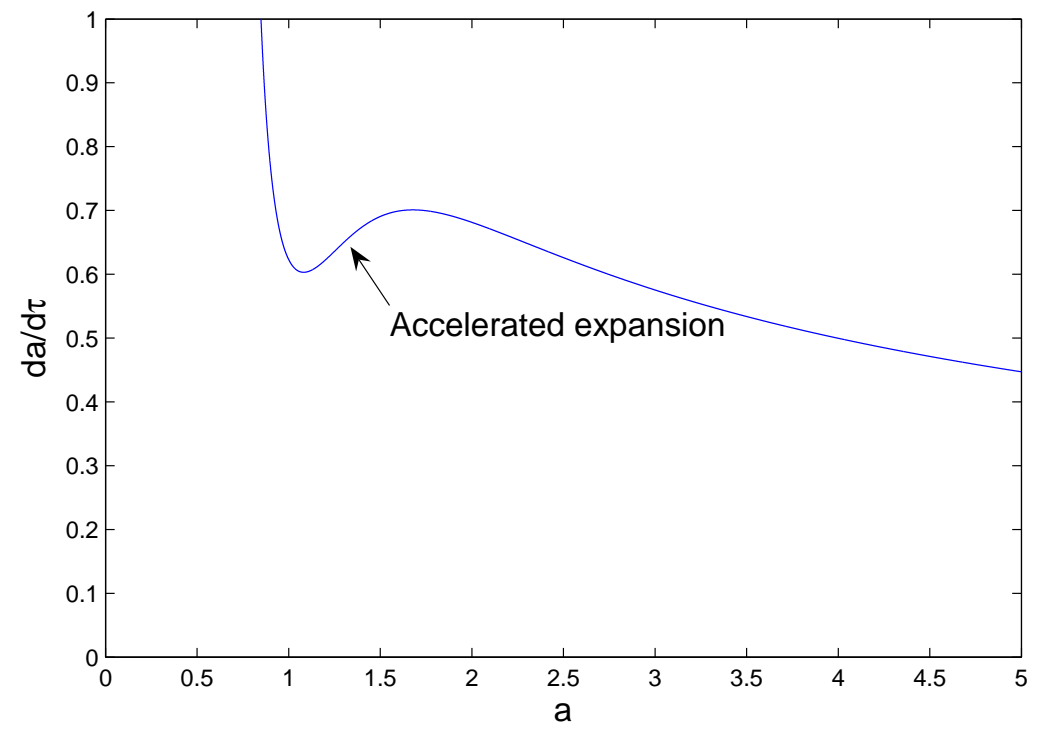

Figure 1: Expansion rate of the universe versus the scale factor $a$ according to the CD theory. The graph is valid both for empty space-time and for a universe filled with an incoherent dust.

in the absence of matter, and, remarkably, one has a sequence of deceleratedaccelerated-decelerated expansion. In fact $\tilde{a}$ from (19) with $\tilde{\rho}=0$ has two extrema corresponding to

$$
\tilde{a}=(12 \pm 6 \sqrt{3})^{1 / 6}
$$

The same result is obtained when matter is present in the form of dust. In that case mass conservation implies

$$
\rho=\rho_{0} \frac{\tilde{a}_{0}^{3}}{\tilde{a}^{3}}
$$

leading to a renormalization of constants not modifying (24). Fig[1 shows the behaviour of the expansion rate as a function of the cosmic scale factor.

The situation is different if we allow for more general forms of matter. For a simple barotropic fluid with an equation of state

$$
\rho c^{2}=w p,
$$

where $0 \leq w \leq 1 / 3$, the conservation laws of thermodynamics imply that

$$
\rho=\rho_{0} \frac{\tilde{a}_{0}^{3(1+w)}}{\tilde{a}^{3(1+w)}} .
$$


In this case the equation for the extrema, from (19), is

$$
\tilde{a}^{3 w}\left(36-24 \tilde{a}^{6}+\tilde{a}^{12}\right) \mathfrak{W}-6 \tilde{a}^{6}(3 w-4)-\tilde{a}^{12}(1+3 w)-36=0
$$

We are left with two free parameters, $w$ and $\mathfrak{W}=\mathcal{W} /\left(c^{2} \tilde{\rho}_{0} \tilde{a}_{0}^{3(1+w)}\right)$, to be determined in order to recover both the observed onset of the accelerated expansion and the age of the universe.

\section{The Newtonian limit}

Since General Relativity satisfies all the Solar system tests, any extension or modification of GR must possess a correct Newtonian limit. In this section we want to compare the theories we have been discussing until now, also on this respect. In practice we expect that, given any material source, the field equations for gravity, in weak field approximation, reduce to the Poisson equation for the potential.

\section{In Vector Ether Theories}

Let us consider the field equations (6) in the static, weak field limit. The way chosen both in [19] and in 20] is to expand both the metric and the vector field around a Minkowski background. At the lowest non-trivial order the approximated line element may be written as follows:

$$
d s^{2}=(1+2 \Phi(x, y, z)) d \tau^{2}-(1-2 \Psi(x, y, z))\left(d x^{2}+d y^{2}+d z^{2}\right),
$$

where $\Phi$ and $\Psi$ are suitable potentials. Since we are in the weak field limit, we shall neglect terms beyond the first order in the potentials; under this assumption the space components of the Æther stress-energy tensor disappear. The spatial components of the Einstein equations reduce then to

$$
\left(\delta_{i j} \nabla^{2}-\partial_{i} \partial_{j}\right)(\Phi-\Psi)=0,
$$

where $i$ and $j$ are space indices ranging from 1 to 3 . Assuming that both $\Psi$ and $\Phi$ vanish at space infinity, (25) implies that $\Psi=\Phi$. Using this result, while combining the linearized 00-th component of the Einstein equations and the vector field equation, we obtain

$$
\vec{\nabla} \cdot\left[\left(2+\frac{16 \pi G}{c^{4}} c_{1} \mathcal{F}^{\prime}\right) \vec{\nabla} \Phi\right]=\frac{8 \pi G}{c^{2}} \rho,
$$

where $\rho$ is the mass density of the matter distribution. In the framework of $\mathbb{E}$ theory, it is simply $\mathcal{F}^{\prime}=1$ and equation (26) becomes

$$
\nabla^{2} \Phi\left(1+\frac{8 \pi G}{c^{4}} c_{1}\right)=\frac{4 \pi G}{c^{2}} \rho
$$


which is indeed a Poisson equation, with an effective gravitational constant

$$
G_{N}=\frac{G}{1+c_{1} \frac{8 \pi G}{c^{4}}} .
$$

The results (27) and (23) can be used to obtain one more constraint on the parameters of the theory, as analyzed in 20] and [21.

In the ZFS version of the theory [19] 5 , the authors are first led to identify the mass scale $M$ with something of the order of $a_{0}$, as we have seen before, in order to recover the MOND limit of the theory. For them it actually is

$$
\mathcal{K}=-c_{1} \frac{(\vec{\nabla} \Phi)^{2}}{M^{2}}
$$

and $c_{1}<0$ in order to avoid ghost-like spin-0 states (see [21]). Now, looking at (26), we see that, in order to recover, at least at the Solar system scale, the Poisson equation, the $\mathcal{F}^{\prime}$ contribution must be small. For these reasons, the authors assume that in the Solar system $(\vec{\nabla} \Phi)^{2} \gg M^{2}$ and expand $\mathcal{F}^{\prime}$ as a series of inverse powers of $\mathcal{K}^{1 / 2}$. At this point, however, the non-linearity due to $\mathcal{F}^{\prime}$ makes the equations very difficult, thus making very hard to draw clear-cut conclusions, as remarked in [25].

\section{In the CD theory}

In the case of the $\mathrm{CD}$ theory, as in the previous section, we have to consider the weak-field limit of the theory, expanding both the metric and the vector field around a background configuration, but now we choose the FRW rather than Minkowski spacetime, because we want to maintain a link between the cosmological and the local solution. The source of the perturbation is assumed to be some local, static matter distribution, superposed to the cosmic one. The details of the whole procedure may be found in [6]; the essentials are outlined in the following.

The perturbed line element is now written as:

$$
d s^{2}=\left(1+h_{0}(x, y, z)\right) c^{2} d t^{2}-a^{2}(t)\left(1+h_{s}(x, y, z)\right)\left(d x^{2}+d y^{2}+d z^{2}\right),
$$

with $h_{0}, h_{s}<<1$.

We expect the flow lines of the cosmic vector field to be perturbed as well, however preserving the norm of the vector, which depends on the presence of the cosmic defect only. Let us write the perturbed vector as $\Upsilon$; its components (first order approximation) will be:

$$
\begin{aligned}
& \Upsilon^{0}=\chi\left(1+f^{0}(x, y, z)\right) \\
& \Upsilon^{i}=\chi f_{s}^{i}(x, y, z),
\end{aligned}
$$

\footnotetext{
${ }^{5}$ Note that, again, the definition of the Lagrangian for the Æther $\mathcal{L}_{U}$ here is $\frac{16 \pi G}{c^{4}}$ times the one found in the cited article.
} 
The perturbations are scaled on the unperturbed norm $\chi$, and we assume that $f^{0}, f_{s}^{i}<<1$ (at least as small as the $h$ 's), and depend on the space coordinates. The time dependence is contained in the scale factor $a(t)$ only. The divergencelessness condition (9) applied to $\Upsilon$ must still hold, because it is broken only at the site of a space-time defect and in this respect nothing has changed, in the sense that no other singularities have been introduced, besides the cosmic one. Eq. (9), at first order in the perturbations, becomes

$$
\vec{\nabla} \cdot \overrightarrow{f_{s}}=0
$$

The invariance of the norm of $\Upsilon$ produces the condition

$$
f^{0}=-\frac{h_{0}}{2} .
$$

The next steps may be summarized as follows: a) introduce the metric (28) in the CD action integral (11) plus matter, then linearize it in the perturbations; b) deduce the field equations for the geometry (the equivalent of the Einstein equations); c) consider that the zero order of the equations is automatically satisfied with the cosmic fluid energy momentum tensor; d) write down the first order equations with the local matter energy momentum tensor $\mathcal{T}_{\mu \nu}$ assumed to be isotropic in space around any given point. You will get:

$$
-e^{-\chi^{2}}\left[\nabla^{2} h_{s}+\chi^{2}\left(\nabla^{2} h_{0}+2 \nabla^{2} h_{s}\right)\right]=\frac{4 \pi G}{c^{4}} \mathcal{T}_{00}
$$

where $\nabla^{2}=\frac{1}{a^{2}}\left(\partial_{x}^{2}+\partial_{y}^{2}+\partial_{z}^{2}\right)$ and $\mathcal{T}_{00}$ is the energy density of the local source. As we know, there is a freedom for the choice of the coordinates, so that the Lorentz gauge can be imposed, leading to $h_{s}=-h_{0}$. The final equation is then

$$
\nabla^{2} h_{0}=\frac{4 \pi}{c^{4}} \frac{G e^{\chi^{2}}}{1+\chi^{2}} \mathcal{T}_{00} .
$$

This equation is the Poisson equation with a renormalized gravitational "constant" slowly changing with time:

$$
G_{*}=\frac{G e^{\chi^{2}}}{1+\chi^{2}} .
$$

The cosmic vector field $\gamma$ does indeed affect the local gravitational field, through its norm $\chi$. This influence is not perceivable on the usual time scales, in the sense that the Newtonian behaviour is fully recovered; however in cosmic times the effective coupling "constant" of gravity, in the Newtonian formalism, slowly changes. Had we started from a Minkowski background, this adiabatic effect would not have been visible, as it happens in the $Æ$ theory where two formally different renormalizations of $G$ are obtained at the cosmic and at the local scale. 


\section{Correspondence between the theories}

VET, as well as the more general SME, and CD are apparently rather different from each other, however, as we shall show here, it is possible to recast the latter in a form which will make it emerge as a special case of the former. The comparison will then be made at the level of the effective action integrals.

Considering the CD action (11) we remark that it could be thought of as being the result of a conformal transformation from some previous appropriate metric. To evidence this interpretation in the present section, we shall mark the entities used in the CD theory with a $\sim$ assuming that $\tilde{g}_{\mu \nu}=e^{2 \omega} g_{\mu \nu}$, being $\omega$ a conformal factor. Let us rewrite (11) accordingly:

$$
S=\frac{1}{2 \kappa c} \int e^{-\chi^{2}} \tilde{R} \sqrt{-\tilde{g}} d^{4} x .
$$

Consistently with the approach we are describing here, the curvature and the square root of the determinant of the metric tensor may be written as

$$
\begin{aligned}
\tilde{R} & =e^{-2 \omega}\left[R-6 g^{\mu \nu} \nabla_{\mu} \nabla_{\nu} \omega-6 g^{\mu \nu}\left(\nabla_{\mu} \omega\right)\left(\nabla_{\nu} \omega\right)\right] \\
\sqrt{-\tilde{g}} & =e^{4 \omega} \sqrt{-g} \\
\tilde{\chi}^{2} & =e^{2 \omega} \chi^{2}
\end{aligned}
$$

If we now choose the conformal factor so that

$$
\chi^{2}=2 \omega e^{-2 \omega},
$$

since of course $e^{2 \omega} e^{-\chi^{2} e^{2 \omega}}=1$, the effective Lagrangian density before the transformation turns out to be

$$
\left[R-6 g^{\mu \nu} \nabla_{\mu} \nabla_{\nu} \omega-6 g^{\mu \nu}\left(\nabla_{\mu} \omega\right)\left(\nabla_{\nu} \omega\right)\right] \sqrt{-g}
$$

Recalling that $\chi^{2}$ is the norm of the vector field $\gamma^{\mu}$, we can rewrite the second and third terms in the square brackets of (40) as explicitly depending on $\gamma$.

The solution to this trascendental equation is the Lambert function, in particular

$$
2 \omega=-W_{k}\left(-\chi^{2}\right) .
$$

The Lambert function $W_{k}(z)$ is a multivalued function of the complex variable $z$ and $k$ is an integer that represents the branch we are looking at. In our case the variable is the norm of the vector field that is time-like: we must restrict to the case in which $z$ is real, let us say $x$, and $x>0$. Furthermore, we want the conformal factor, as well as the Lambert function, to be real, so that we consider only the case in which the argument is greater than $-1 / e$. But in our solution the argument of the Lambert function is $-\chi^{2}$, that is, the norm of the vector filed can change only between 0 and $1 / e$. In order to enhance this range we can solve the subsequent equation, instead of 39 ,

$$
e^{2 \omega} e^{-\chi^{2} e^{2 \omega}}=\text { const } \Rightarrow \chi^{2} e^{2 \omega}-2 \omega=c .
$$


This simply means that we are changing the value of the dimensional constant in front of the action integral. The solution, now, is

$$
2 \omega=c-W_{k}\left(-e^{c} \chi^{2}\right)
$$

and the range of the variable for which the Lambert function is real. To enhance it we have to choose $c<1$.

But let us note that in the range we are interested in the Lambert function is two-valued. For $W(x) \geq-1$ the function is denoted $W_{0}(x)$, or simply $W(x)$, and is called the principal branch; for $W(x) \leq-1$ the function is denoted $W_{-1}(x)$.

If we look for the principal branch, a Taylor series can be found but due to the singularity at $x=-1 / e$ the series converges for $|x|<1 / e$. The series is

$$
W(x)=\sum_{n=1}^{\infty} \frac{(-n)^{n-1}}{n !} x^{n}=x-x^{2}+\frac{3}{2} x^{3}+\ldots
$$

Rewriting the solution in terms of this series, and remebering that the action integral of VET is fourth order in the fields, we can drop all terms beyond second order, since the variable $x$ corresponds to the norm of the vector field, that is second order in the field.

Now, we are left only with the terms that appear in $\tilde{R}$. Apart from the first term, that is the Ricci scalar in terms of the old metric, there are two additional terms. Let us look at the first:

$$
\begin{aligned}
g^{a b} \nabla_{a} \nabla_{b} \omega & \sim g^{a b} \nabla_{a} \nabla_{b}\left(c+e^{c} \chi^{2}-e^{2 c} \chi^{2} \chi^{2}\right) \\
& =2 e^{c} g^{a b} \nabla_{a}\left(\gamma^{c} \nabla_{b} \gamma_{c}\right)-e^{2 c} g^{a b} \nabla_{a} \nabla_{b}\left(\chi^{2} \chi^{2}\right) \\
& =2 e^{c} g^{a b} \nabla_{a}\left(\gamma^{c} \nabla_{b} \gamma_{c}\right)-2 e^{2 c} g^{a b} \nabla_{a}\left(\chi^{2} \nabla_{b} \chi^{2}\right)
\end{aligned}
$$

where both terms are divergence, that is they reduce to surface terms when integrating. We are left only with the second term in (36), in which, in order to obtain terms up to fourth order, reduce to the following one:

$$
\begin{aligned}
g^{a b}\left(\nabla_{a} \omega\right)\left(\nabla_{b} \omega\right) & \sim g^{a b} \nabla_{a}\left(e^{c} \chi^{2}\right) \nabla_{b}\left(e^{c} \chi^{2}\right) \\
& =e^{2 c} g^{a b}\left(2 \gamma^{c} \nabla_{a} \gamma_{c}\right)\left(2 \gamma^{d} \nabla_{b} \gamma_{d}\right)=4 e^{2 c} g^{a b} \gamma^{c} \gamma^{d} \nabla_{a} \gamma_{c} \nabla_{b}(A 3)
\end{aligned}
$$

Our effective Lagrangian density is now:

$$
\left(R-6 e^{2 c} g^{\mu \nu} \gamma^{\alpha} \gamma^{\beta} \nabla_{\mu} \gamma_{\alpha} \nabla_{\nu} \gamma_{\beta}\right) \sqrt{-g}
$$

What here is called $\gamma$, in the $\mathrm{VE}$ theories is the $U$ vector, so that comparing (44) with the Lagrangian density in (2) we see that the CD theory corresponds to a VE theory with the only $c_{6}$ coefficient differing from 0 . Actually it is $c_{6}=-6 e^{2 c}$.

As we wrote in section (II), VET may be considered as special cases of the SME theory so that a direct comparison to that theory is appropriate. A simple example of a cosmic vector field is the so called "bumblebee" vector field illustrated in appendix B of ref.[15]. The bumblebee field is indeed a 
timelike vector $B$ dynamically depending on a suitable potential in a torsionless spacetime. Writing $B_{\mu \nu}=\left(\nabla_{\mu} B_{\nu}-\nabla_{\nu} B_{\mu}\right)$, the Lagrangian density is assumed to be

$$
\mathcal{L}_{B}=\frac{\xi}{2 \kappa} B^{\mu} B^{\nu} R_{\mu \nu}-\frac{1}{4} B^{\mu \nu} B_{\mu \nu}-V\left(B^{\mu} B_{\mu} \pm b^{2}\right)
$$

where $\xi$ is a parameter and $V(x)$ is a scalar potential; $b$ sets the position of the minimum of the potential. Comparing (45) with (22) and (3) we see that the former corresponds to the latter for peculiar combinations of the $c_{i}$ 's (of course to see this one must express $R_{\mu \nu}$ in terms of the $g_{\mu \nu}$ 's and their derivatives, then making some integrals by parts in the action). In particular the "bumblebee" model is recovered when $c_{1}=-1 / 2, c_{3}=1 / 2-c_{2}$.

\section{Conclusion}

We have been analyzing in parallel the CD theory, on one side, and the VE theories (and especially the Einstein Æther theory) on the other. Both (groups of) theories are based on the presence of a cosmic timelike vector field, and may be thought of as special cases of the SME theory.

The VET contain a big number of adjustable parameters, and, in the ZFS case, also a free function. Various ways to exploit this wide freedom allow for different approaches and lead to different conclusions. In fact, rather than a global scenario, a number of specific, not completely consistent pictures emerge. For instance the accelerated expansion is present in one version, and not in others; the gravitational coupling constant has formally different limits in the cosmic and in the Newtonian limit. Furthermore the physical meaning of the majority of the free parameters of the theory is unclear.

In the CD theory only one free parameter exists in the description of space time: a sort of global scale constant, to be determined on the basis of the observed behaviour of the universe. One more parameter appears when considering the coupling between matter and spacetime. The theory accounts for the accelerated expansion and possesses a Newtonian limit with a renormalized gravitational "constant" slowly changing in time.

Apart from the details, an important difference between VET and CD is in the embedding paradigm. In the case of VET we are in the mathematical framework of vector-tensor field theories, and the hypothesized vector field, as well as many parameters, lack a physical motivation other than the final result. In the case of CD the paradigm is based on some analogies with problems of the physics we already know, and the vector field is thought of as the consequence of the strain induced in a four-dimensional medium (spacetime) by the presence of a defect, in the sense of the elasticity theory. This paradigm makes the theory very compact, minimizing the number of adjustable coefficients and making the comparison with observation easier or, at least, the conclusions sharper.

The fact that the real main difference is in the interpretation paradigms appears clearly when, as we did in Sec. (VI), we show that CD may be looked at as to a special case of VET. However, had we gone from the Vector Æther 
theory to the Cosmic Defect, the corresponding peculiar choice of the parameters of VET would have appeared to be completely arbitrary. On the contrary the approach used for CD provides a consistent interpretation scheme, which in the end is shown to be mathematically equivalent to one specific Æther theory.

For these reasons we think that the CD paradigm can be fruitfully exploited again for a deeper understanding of the evolution of our universe.

\section{References}

[1] S. M. Carroll in Carnegie Observatories Astrophysics series, Vol.2: Measuring and Modelling the Universe, W. L. Freedman Ed., Cambridge Univ. Press, (Cambridge 2003).

[2] Sean M. Carroll, The Cosmological Constant, Living Rev. Relativity, (2001), 1. URL (http://www.livingreviews.org/lrr-2001-1)

[3] Peebles, P.J.E. and Ratra, BV., The Cosmological Constant and Dark Energy, Rev. Mod. Phys. 75, 559-606 (2003).

[4] D. Wands, Extended gravity theories and the Einstein Hilbert action, Class. Quant. Grav. 11, 269 (1994).

[5] E. E. Flanagan, Higher order gravity theories and scalar tensor theories, Class. Quant. Grav. 21, 417 (2003).

[6] A. Tartaglia and M. Capone, Space time as a continuum with a point defect, gr-qc/0601033v5 (2006).

[7] C. Eling, T. Jacobson and D. Mattingly, Einstein-Ether theory, in "Deserfest: A Celebration Of The Life And Works Of Stanley Deser", J. T. Liu, M. J. Duff, K. S. Stelle, R. P. Woodard eds., World Scientific, Singapore (2006), p. 163; gr-qc/0410001.

[8] V.A. Kostelecký and S. Samuel, Phenomenological Gravitational Constraints on String and Higher-Dimensional Theories Phys. Rev. Lett. 63, 224 (1989); Gravitational phenomenology in higher-dimensional theories and strings Phys. Rev. D 40, 1886 (1989).

[9] S.M. Carroll, J.A. Harvey, V.A. Kostelecký, C.D. Lane and T. Okamoto, Noncommutative Field Theory and Lorentz Violation Phys. Rev. Lett. 87, 141601 (2001); see also references in [8].

[10] T. Jacobson and D. Mattingly, Gravity with a dynamical preferred reference frame Phys. Rev. D 64, 024028 (2001).

[11] C. Will and K. Nordtvedt Conservation laws and preferred frames in gravity, Astrop.J.177, 757 (1972); R.W. Hellings and K. Nordtvedt, Vector metric theory of gravity Phys. Rev. D 7, 3593 (1973). 
[12] P.G. Ferreira, B.M. Gripaios, R. Saffari and T.G. Zlosnik, The cosmology of a universe with spontaneously-broken Lorentz symmetry Phys. Rev. D 75, 044014 (2007).

[13] T. G. Zlosnik, P. G. Ferreira, G. D. Starkman, The Vector-Tensor nature of Bekenstein's relativistic theory of Modified Gravity, Phys. Rev. D 74, 044037 (2006).

[14] B.M. Gripaios, Modified gravity via spontaneous symmetry breaking J.High Energy Phys. 10 (2004) 069.

[15] V.A. Kostelecký, Gravity, Lorentz violation and the standard model, Phys. Rev. D 69, 105009 (2004).

[16] D. Colladay and V.A. Kostelecký, CPT violation and the standard model, Phys. Rev. D 55, 6760 (1997); Lorentz-violating extension of the standard model, Phys. Rev. D 58, 116002 (1998).

[17] Y. Nambu, Quantum electrodynamics in nonlinear gauge, Prog. Theor. Phys. Suppl. Extra 190 (1968).

[18] M.A. Clayton, Causality, shocks and instabilities in vector field models of Lorentz symmetry breaking, arXiv: gr-qc/0104103.

[19] T.G. Zlosnik, P.G. Ferreira, G.D. Starkman, Modifying gravity with the Ether: an alternative to dark matter Phys. Rev. D 75, 044017 (2007).

[20] S.M. Carroll and E.A. Lim, Lorentz-violating vector field slow the universe down Phys.Rev. D 70, 123525 (2004).

[21] E.A. Lim, Can we see Lorentz-violating vector field in the CMB? Phys. Rev. D 71, 063504 (2005).

[22] C. Eling and T. Jacobson, Two-dimensional gravity with a dynamical aether, Phys. Rev. D 74, 084027 (2006).

[23] V. Volterra, Ann.Éc.Norm.Sup.24, 401 (1907).

[24] M. Milgrom, A modification of the Newtonian dynamics as a possible alternative to the hidden mass hypothesis, Ap.J. 270, 365 (1983); A modification of the Newtonian dynamics: implication for galaxies, Ap.J. 270, 371 (1983); A modification of the Newtonian dynamics: implication for galaxy systems, Ap.J. 270, 374 (1983).

[25] J. Bekenstein and J. MagueijoD, Modified Newtonian Dynamics habitats within the Solar System, Phys. Rev. D 73 103513,(2006). 